Der Anfidt, Doß folde Entwáfferungen felyr zu überlegen feien.

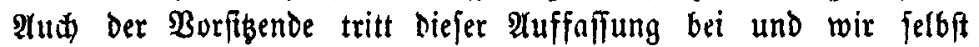
glauben, Daßj man in Diejer Begiegung leiđjt zu weit geken fonn und audi bereitz an mandjen Drten zu weit gegangen ift, wenn

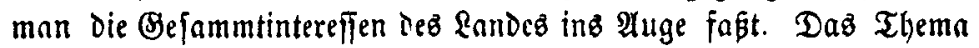

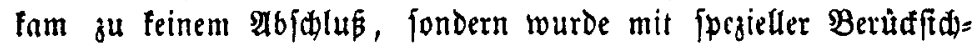
tigung Der Berbältniffe in Şarz nod etwas mebr geipalten und Jur Demnädftigen Beantwortung Den Bereinşmitgledern empfohlen. - Wir find Der Meberzeugung, daß es fíf hier um einen Ssegenfand von grofer Trngmeite handelt, Der verdiente einmal in einer sllgemeinen forftberjammlung gründliक bejprod)en zu werden.

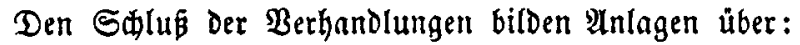

1) Die Forfte Der Braffinaft Frallenftein.

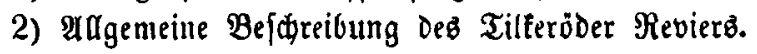

3) Befareibung Der $\mathfrak{B a l D e r c u r f i o n e n}$ am 2. und 3. Sept. 1867.

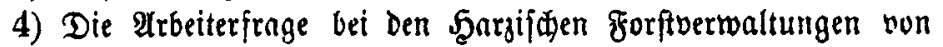
Gorftmeifter $R$ ett fit a $d$ t.

Berme würden wir bei Den yorliegenden intereffanten Berballd: lungen Deß Şarger Forftwereing nod) länger verweilen, weln eछ uns

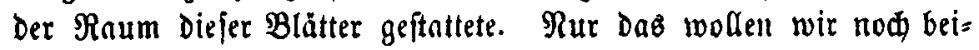

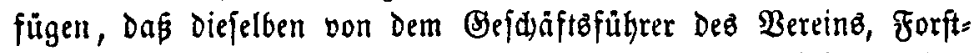
meifter Beling in Seejen, wiederholt jefr gut redigirt worden

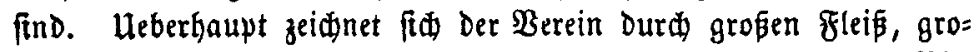
Eez. Streben und bohe Reiftungsfähigfeit aub. Die einzelnen Mit= glieder fommen nidt, wie man Das anderwärts öfters findet, uns vorbereitet und bemegen fid Daher nidt auf abgebrofdenen Bemein= pläb̨en, fondern fie bringen forgfältige Beobadtungen, Zahlen und Thatjaden mit auf die Berfammlungen, wohl miffend, Dás nur

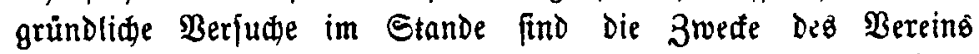
Dauerno zu fördern.

18.

\title{
№.. 13.
}

Berhandlungen Ded badif

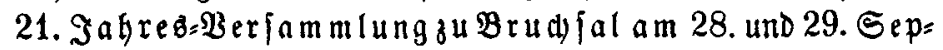
tember 1868. Freiburg, $\mathfrak{W}$ angler 1868. 83 Seiten in gr. 8 . Der badijaje Forftwerein, welder 1839 gegründet worden ift 
und jebt 192 Mitglieder jabli, bieft frine 21. Snkrebuerjammlung am 28. und 29. Septemler 1868 in Brudfal, Dem Sinotenpunfte Der badifden und württembergifđen Sgnuptbaly. Sie war von 65 Theilnehmern bejưt, unter benen fît) fämmtlide now lebende Gründer Des Bereinez, fonie eine große 3 nhl feiner getreueften Slieder und ein tüdtiger Mittels und જungmugh befallden. Die Mebrgubl bildeten Die Badener, aber audi unjere werthen $\mathfrak{R a d b a r n , ~}$

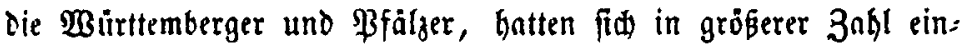
gefunden, und son bn und Dort befudten uns Eingelne, alte bes währte Freunde und neuse Greunde, welde hod willtomnen waren. Die Berjammlung wöre nuв Baden felbft zahlreidjer bejudjt wor= Den, wern nidgt in jenten Tagen in vielen $\mathfrak{R} a n d e$ gegenden die

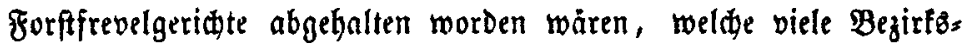

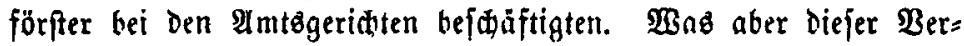
jammlung eine größere Bedeutung gab, war bie Inve fenbeit bon

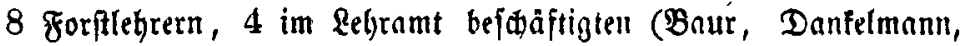
Sduberg, Bonfaufen) und 4 in Den preftip(den Dien zurüfgetre: tenen (b. Bredt, Fifdhađ, Fromann, Sdjott non Sdottenftein).

Radoem einige bäuslide Angelegentheiten geordnet waren, und Der Berein fid) für bie 3 näditen Berjammlungen einen neuen

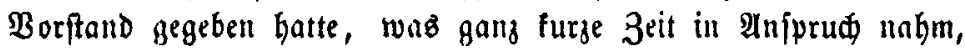
swurie mit Der Berhalldlung der programmmäajigen Begenftảnde begonnen.

Der erfte Begenftand betrifft eime Der brennenden Fragen unferer Beit, Die Streufrage, in folgender Faffung:

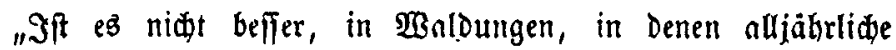
"Streunugungen niöt abgeftellt werden tönnen, Diefe nur in Den

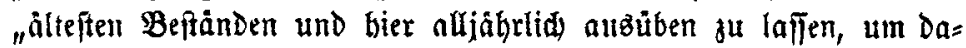

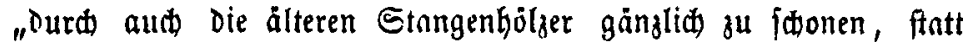
"foldhe in allen gefeslid) geöffneten Beftänden abwedjelnd eintreten "ž Laffen?"

Die Frage umfaßte hiernad nur eitren Theil Der Streufrage. Der Referent, Serr Doerforftrath Seidel, entwicfelte ben Eał, Dnp ein= ober zmeijährige Sdonungşzeiten nuklob frien und hảufigez

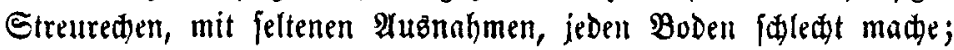




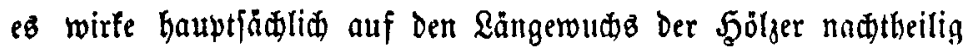
ein, während Der હđaden bei beendigtem \&ängewuđz minder yroß jei. Daker jodade mant in alten Beftinden, wenn in ignen die

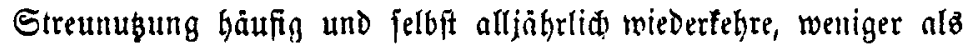
in jüngeren bei auğfę̧enver Streunuģung.

Dieje Anfidit wurde von Şerrn \$rofeffor Bontaulfen be=

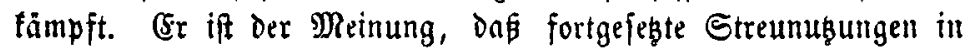

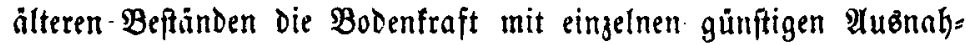
men rafd erfdöpfen, namentlid im Riedermaldbetriebe, Da Derjelbe Die Bodenfraft weniger zu erhalten sermöge, als der Şodwald.

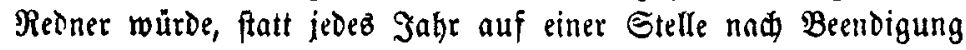
Deg borkerridenden Şöhenmudjez Streu zu redien, einen minde= ftens zweijährigen Streuturnuछ einfübren, und daz Streufammeln 5-6 Jabre vor ber Beriüngung gang einftellen. Er glaubt ủbri: genz, Daß man mit jolden untergeoroneten Mitteln bie Şauptfrage

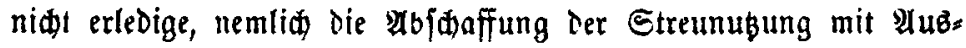

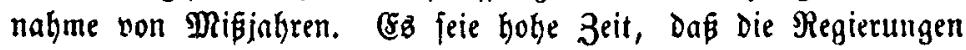
Die Şandidube augiethen und die Etreufrage fräftig in bie Şand nehmen.

Şerr Oberförfter Şeî́ ift für eine, $2 / 3$ ber Ummtriebżzit um= faffende હdyonung und eine 10 jägrige $\mathfrak{B}$ orhege vor Der Berjüngung.

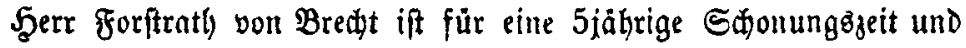

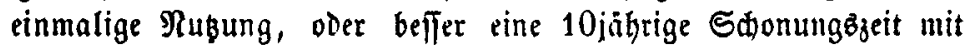
2 aufeinanter folgenden Ruţungen. Şerr Forftmeifter bon Słult

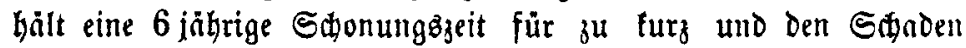

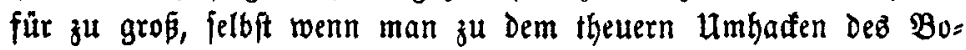
Denz greife.

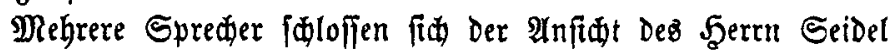
an und es wurden now verfoiledene (Erfahrungen mitgetbeilt, wie die Streunukgung, wo fie zur Zeit beftethen müffe, am wenigiten

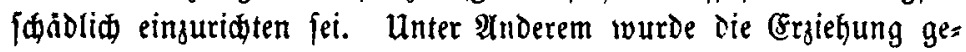

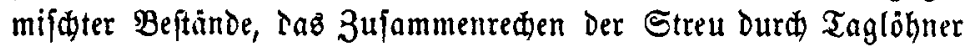

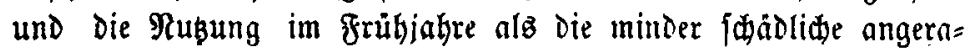
then. atue Stimmen vereinigten fid aber Dahin, Das bie Bejeg= gebung fiti) des vorhandenen Nothfitandes annetgen uno denjelben 


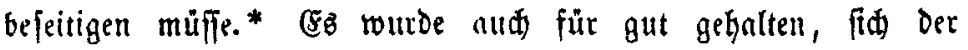
Preffe zu bedienten, um iaż Bolf iber bie Ende zu beleftren.

Die groeite $z$ rage lautet:

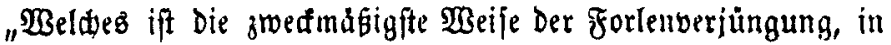

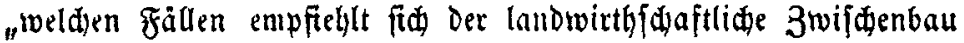
"und wie wird bierkei berfahren?"

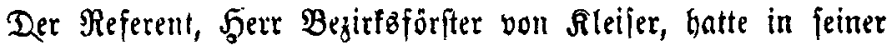
Auffaffung Der Frage Die Forlenwaldungen in Den TilubialböDen

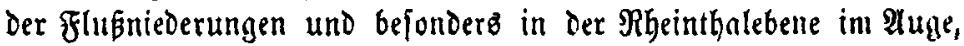
beseidnete für biefe Dertliđfeiten Die natürliđe Berjủıgung als un= zureiđend und in jeder Begiegung ungenigend, und rieth bie tünft=

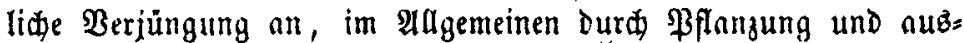

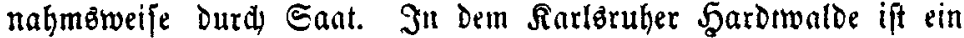

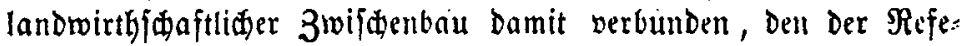
rent genau befdreibt, er will aber ibn nur als Mittel zum 3wede Des Sूolzanbaues betrodten, auf müglichtit furze Zeitbauer befdrän= ten und nur auf ben fräftigeren Sandböden zulaffen. Şier hgit

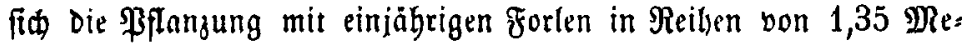
ter Entfernung und 0,6 - Meter 2 bftand der Pflangen am beftent bewäbrt, es rerden bollfommente Beftinde ergielt und die Erträge

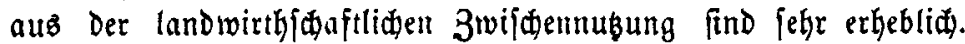
Namentlid wird aud Der fräftige \&ängemucts Der auf Dem ge= loderten Boden erzogenten Forlenbeftande herborgehoben.

Die Erfabrungen beş Şerrn von Fleijer fanden vieljeitigen BBiberjprut) von Mitgliedern Der Berfammlung, welde auz andes ren Begenden "Famen. Theilz wurbe Der natürliđen Berjüngung in ber[jiedenen formen baz Bort gereiet, theilo der Innowirth=

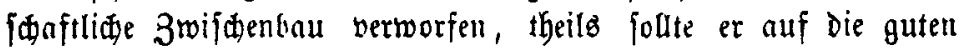
Böben bejarảnft werben, einige enipfablen bie Saat, andere bie

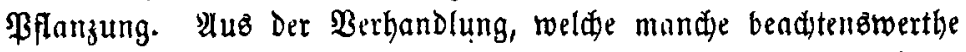

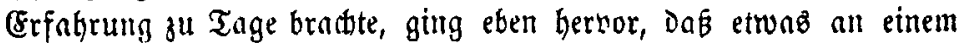
Drte gut, am anderen mittelmäßig und am Dritten jकletht fein fann. WBe in anderen Fåalen bedingen die örtliden Berhältniffe

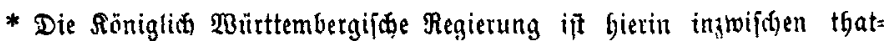
lräftig rurnngegangerr.

1869. 
Die $\mathfrak{A r t}$ Der Beriüngung, fowie ifre mandfaltigen Formen; bie

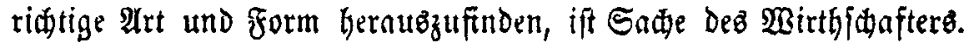

Die Dritte Frage tautet:

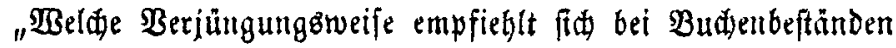
"auf Dem geringeren Eandboden Der Evene uno Borberge, worin "eime natürlidse Beriüngung beabfithrigt ift?"

Şerr Bejirfzförfter (Samer, welder Die Einleitung übernommen Gatte, empfahl Borbereitungshiebe mit guter Bodenloderung Durd, Furzbafen und wenn nöthig mit Eirlaat von Bumeln, fowie bie (Einnifinung Der Forle. Alugenideinlid hatte Die Frage eine fu

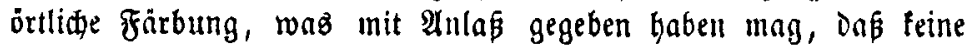
weiteren Mittheilungen gemacht wurben.

Die bierte grage lautet:

„Welde Erfakrungen liegen vor über Das Meberbalten und

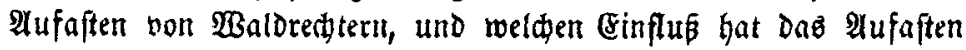
"Derfelben?"

Şerr ßrofefior Sduberg hatte die Einleitung übernommen.

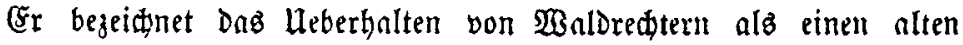
Deutiden Braut) und nidt als eine (Erfindung Der Reujeit. Diejes

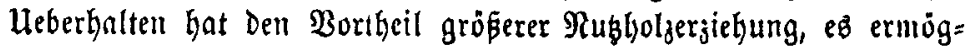

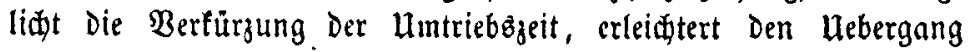
won ber Bremnholzwirthfiaft in die Rughfolgwirthfdaft und von

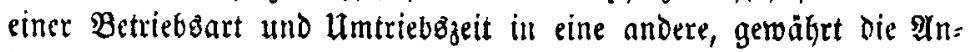
fammlung wertlgoller Borräthe, auf welde man jedergeit greifen fann uno bringt nerfoliedene wirtbidaftlide Borthete, welden bie

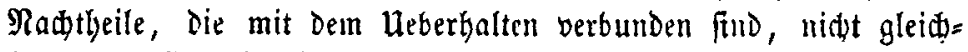
fommen. Ez wird im allgemeinen empfoblen, nur auf günftigen Standorten und in gejdurbter Rage $\mathfrak{B a l D r e d t e r}$ übergubalten, Die rebten Şolzarten und Stämme aubjumäblen, und folde mehr an

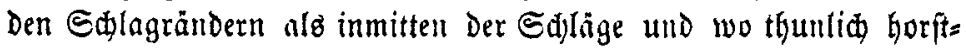
weife überzutgalten.

Bon Den Şolzarten werden unter Den Raubhölzern empfohlen: bie (Eidje in erfter Reibe, fooann ulme, Eifie und shthorn, weniger die Sgrinbuche und am wenigften die Rothbudse, ginde u. f. w.; von Den Radelbölzern taugen am beften Die Forle und Beiftanne, 
weniger bie fidfte, und Die Rärdje gar nidjt. Se günftiger ber Standort ift, befto mehr $\mathfrak{B a l d r e c t e r ~ f o ̈ n n e n ~ u ̈ b e r g e h a l t e n ~ w e r d e n . ~}$

Das find Die Brundregeln des Ueberthaltens, aber es find now viele Fragen Durüber auf Dem $\mathfrak{x}$ ege Der Erfahrung abz̆l= tlären.

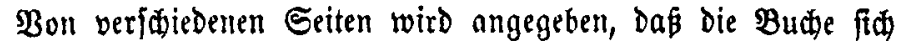

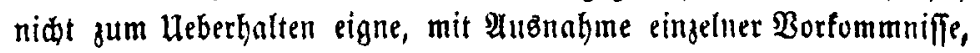
yon Denen namentlid Waerr Forftmeifter Tanfelmann eines_ermäbnt. Bezüglid Der anderen Şotzarten wurden feine abweidemben Er: fahrungen mitgetheilt. Sn Betreff Der Gidije wurde bon Şern Forftrath v. Bredt herborgetoben, Daß fie zum $\mathfrak{W a l d r e d t e r ~ e r z o g e n ~}$ werden müffe. Bon verføiedenen Eeiten wurde das Uleberhalten Der Eidie und $\mathfrak{B}$ eistanne in Şorften mo ganzen Bruppen bebor= wortet. Saerr Forftwraftifant $\mathfrak{y}$. Teuffel bemerft, Daß er bei unter= fudungell all freiftehenden $\mathfrak{B}$ eiffannen im Babener Stadtwalde an alten Stämmen faft teinen Zuwade mehr gefunden habe, bei jüngeren aber bis zit 10 srozenten Der übergehaltenen Marfe.

Sehr belebi war. Die Berbandlung über das Alufaftell und เซลอ Damit zufammenkängt, nomentlid wie weit Die Afufafturg zu gehen babe, welde Bortheile und Mațtheile fie gewähre, ob fie vor, während oder nad der Saftzeit gefdehen folle, hart am Stamm oder nimt, und weldic $\mathfrak{B e r f}$ geuge Die geeignetftell feien. Dod

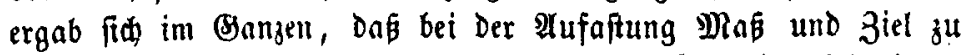

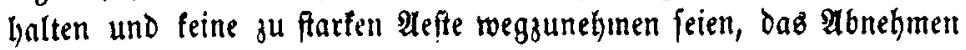

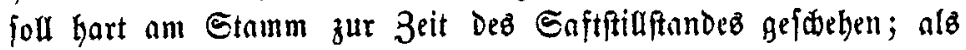

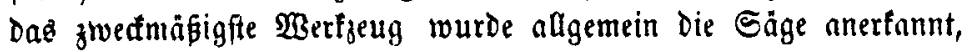

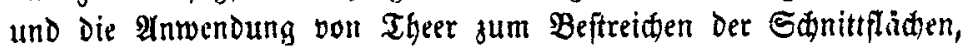

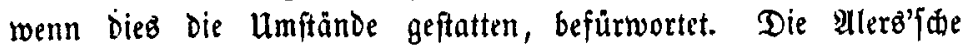
fogennnnte Flügelfige, iber welde in Der neueren 3 eit fo viel gefdrieben worben ift, und bie man befper Stangenfäge nemen wurde, wurde theils empfohlen, theils ihr hoher Breiz bealltandet, ${ }^{*}$

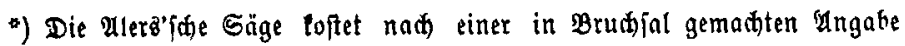
4 Tgaler, nach den gebructen Haltübigungen nber mit 3ugeför 5 Thaler 10 Srofden, von einem Sdiweiger Igenten wurbe fie mir ju 22 Frant $=5$ Thaler 26 srofón angeboten, in Manngeim toftet fie 9 fl. $54 \mathrm{tr}$. ober 5 Ibaler 20 (strojden. 
audy war bon anderen Sägent die Rede, theils auf Etangen, theilb yon Şand zu gebrauthert.

Die fünfte grage ift Die fảndige uno betrifft Mittheilungen

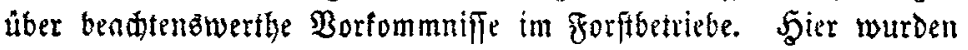
Mittleilungen über sine meue $\mathfrak{A}$ rt non Sprengidjrauben gemadt,

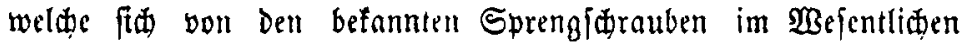

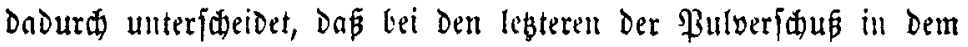
зu fprengenden Stofe fibt, hier aber in Dem (Stejdobe jelth *).

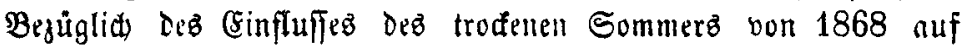
Die Fulturen wurden verjuiebene Mittheilungen gemnat, meifteno jebre ungünftige, am befen bntten fid Ballen: und Şủgelpflanzungen

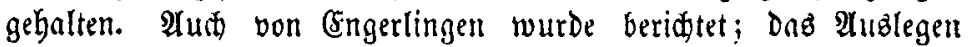

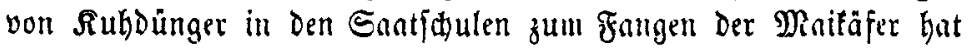
fid) wirfungslos gezeigt. AHe ber Mainebene wurde von einem

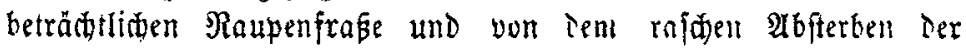
Raupen Mittbeilung gemarit. - Der Eamenerwad) zourde non

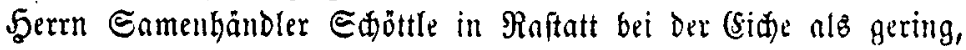

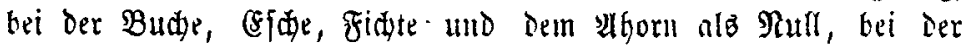
Forle alb ein laalber angegeben.

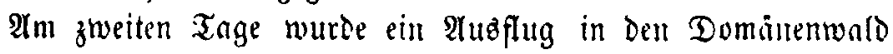
\&ushard gemadt, Der in Der Rheinebene liegt und von weldem

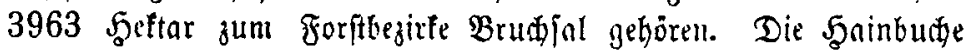
ift hier bie herrfhento $\mathfrak{S g l j}_{j}$ art, gemifat mit Buateu und (siden

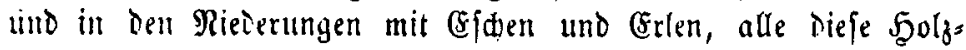
arten auf gutem Boden in frobem und üppigem $\mathfrak{B a d a g t h u m . ~}$

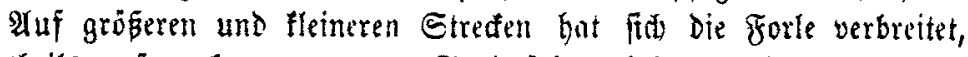
theilz auf trofenen mageren Sandrüfen, theilz alz Grolge ier ver=

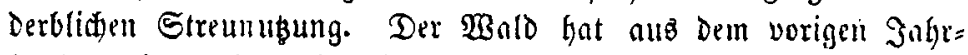
bundert cine vollitäntige, fehr regelnäßige Jageneintheilung, welde

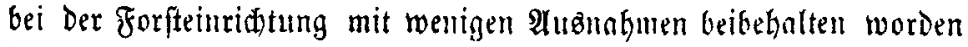
iff; die einjelnen Gagen ober albthetlungen enthalten Durdjignittlid

*) In mebreren fürflith fürftenbergifthen Forften rerden Berfuche mit

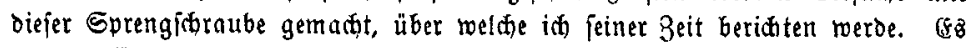
if mebrfältig angegeben morden, dab bie Sprengforarabe nidst treu jet, dann if breitet iff. 
212 Morgen $=76,32$ Sूeftar und find na西 Den Dermaligen $2 \mathbb{2} n=$

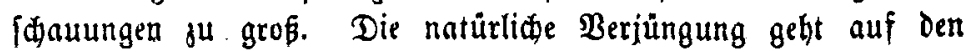
befferen Böden leitet vor fich und wird nach dell dermalen herridens Den geläuterten Grundjäß̨en betrieben, eine nähere Be[đreibung Der Birthfdaft würde aber bie Bretlyen diefed Beridtes überjureiten.

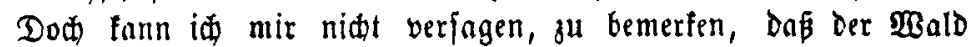

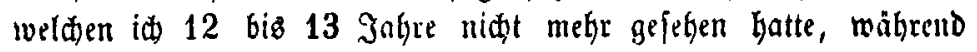
Derfelben bedeutend herangemaden iff. Anterdings waren ia und Dort Die iølimmen $\mathfrak{B}$ irfungen Der Streunukung, erlaubter und unerloulter, felys fithtbar. Iin Sanzen madst die Berwaltung

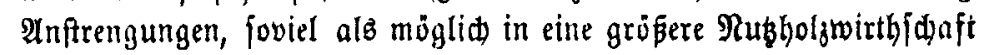
überzugehen.

Infere Brudfjaler Berfammlung hat wohl Den gröbten Theil unjerer Benoffen und Freunde befriedigt, wozu Das freundlide, mit Danf emipfundene Entgegenfommen der Stadt, ihrer Behörden und Betwohner nidt twenig beigetragen lyat. Ter Forftmann füblt fid bald beimifa, wo es ihm nefält, und ber genoffenfitaftlite ein:

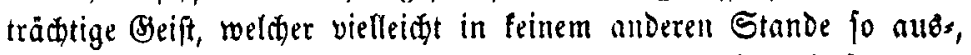
geprảgt ift, als in jenem ber Forftmảnner, läat if̧re 3ujammen= fünfte neben Deen, wns fie für sie $\mathfrak{B}$ Biffenfadaft leiften oder leiftent follen, als eithe art grober zamilienfepte erịteinen, auf welde man fid, wenn man Dnz redite Foer und Den rediten Sinn bafür hat, lange vorker freut. Darum ifte vorauszujeten, Dof wir uns, fojern wir fönnen, aud ferner zujammenfuben werden, Die Senoffen Dez $\mathfrak{B}$ ereines und bie wertben Greunde, weldje mit umb gleide 3weate werfolgen.

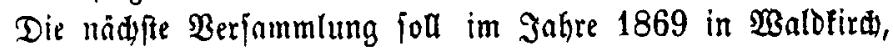

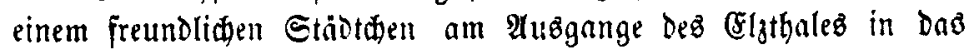
Rkeinthal, 2 Etumten ron Ereiburg im Breiagau gelegen, ftatt= finden, noralisfictlich im Nonat September. Der Drt ift von Der

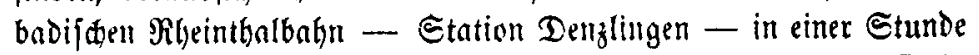

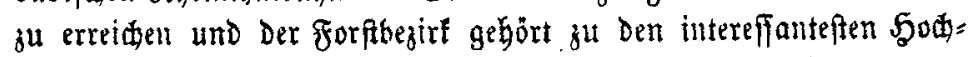
gebirgbforiten Dez fübliden Edmargwaldes, in weldem mant

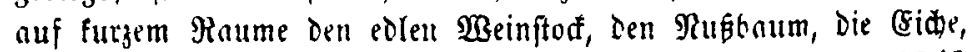

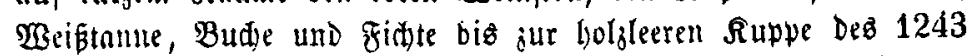
Meter hoben Randel trifft. Die auber Dem ftändigen Thema 
zur Bertandlung fommenden Begenftände find: Máfregeln jur Şebung Des Reinertrags Der $\mathfrak{W a ̈ l}$ er und in $\mathfrak{B e r b i n d u n g ~ D a m i t ~}$

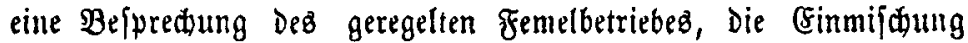

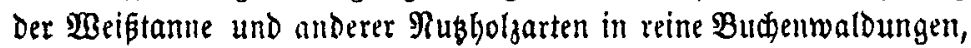
Die Umwandung Der Reutberge in Eidenfiallwaldungen, Das Rapitel Der Şügelpflanzungen und anderer neterer אulturmetboden. (56 fehlt aljo nidit an Stoff ju frudtbaren Bejprectungen und nidit

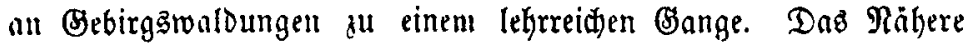
wird feiner Zeit Dnä ßrogramm bringen. $\mathfrak{A} u f$ ein fröhlides Biederjelyen, werthe Greunde und Bentuln, in Maldfirdy!

Donauejwingen.

lath.

\section{$\mathcal{A} \mathfrak{n} 3 \mathfrak{e} \mathfrak{i} \mathfrak{g} \mathfrak{e}$ n.}

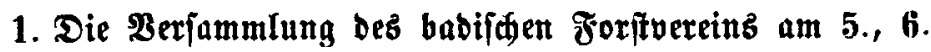
und 7. September 1869 in $\mathfrak{B a l b l i x h}$,

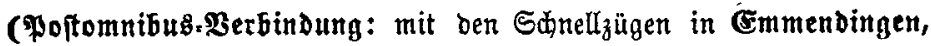
mit ben übrigen ßügen in Denţ

woz̧u Die Bereinzimitglieber, alle Goritminner und Frenute Der Gorftuirthidaft freundlids eingelnoen werden.

\section{Tyagesoromutitg:}

Sonntag ben 5. September, Radmittags:

Snmmlung in (Safthof jum Rỏwen (\$oft), wofelbit Die Ein= forreibung ftatfintet und Die Bobnungen nad)gewiefen werdent.

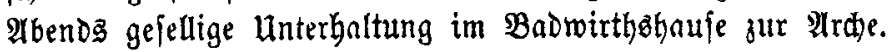

Montag ben 6. September:

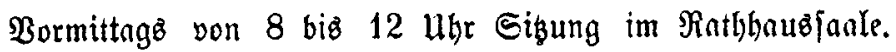
Bereins̄nigelegenbeiten und Berbandlung tiker folgente Fragen:

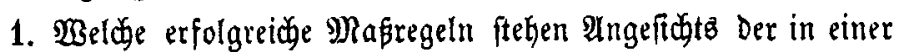
beutliden $\mathfrak{B}$ Bnd

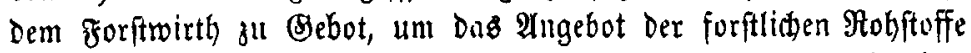

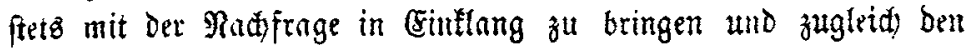
$\mathfrak{B a l}$ oreinertrag za heben? 